\title{
Riga-Fede Disease (Dentitia Praecox): Report of Two Cases with Literature Review
}

\begin{abstract}
Mehta A*, Chaudhary S, Chaitra TR and Sinha A Department of Pedodontics and Preventive Dentistry, Kothiwal Dental College and Research Centre, India *Corresponding author: Mehta A, Department of Pedodontics and Preventive Dentistry, Kothiwal Dental College and Research Centre, Moradabad-244001, India
\end{abstract}

Received: February 13, 2017; Accepted: April 06, 2017; Published: April 28, 2017

\begin{abstract}
The term Riga-Fede disease has been used to describe chronic traumatic ulceration that occurs on the ventral surface of the tongue by natal and neonatal teeth in infants. In order to be termed as Riga-Fede disease, this lesion should be present in a child less than two years of age. Over the age of two years, the term oral traumatic granuloma is used. It is important for the dental professional to recognize the causative agent for Riga-Fede's disease so that a proper assessment, diagnosis and treatment can be performed. This case report presents the occurrence of Riga-Fede Disease and the treatment approach utilized in the two cases.

Keywords: Riga-Fede Disease; Traumatic ulceration; Traumatic granuloma; Natal teeth; Neonatal teeth
\end{abstract}

\section{Introduction}

Riga-Fede disease is a term used to describe traumatic ulceration occurring on the ventral surface of the tongue in neonates and infants [1]. This lesion was first described by Antonio Riga in 1881 and subsequent histological studies were performed by Francesco Fede in 1890, which led to the lesion being termed as Riga-Fede disease [2].

It is commonly caused by mechanical trauma from natal, neo-natal or primary lower incisors and rarely may be the initial presentation of a neurological disorder [1,3]. Various terms have been used to describe this lesion, like Riga's disease, Riga-Fede's disease, sublingual ulcer, sublingual granuloma, traumatic sublingual ulceration, eosinophilic granuloma, traumatic eosinophilic ulceration of the tongue and oral mucosa, sublingual fibrogranuloma, sublingual growth in infants and traumatic atrophic glossitis [4,5].

The lesion initially presents as an ulcerated area on the ventral surface of the tongue and with repeated trauma, it may progress to an enlarged, fibrous mass giving the appearance of an ulcerative granuloma [6]. The pain associated with this oral lesion leads to dehydration and feeding difficulties, which in turn may pose potential risks to infants due to nutritional deficiencies [7]. If the child is immunocompromised, then the potential for infection can add to the complications of the disease. In order to be termed as Riga-Fede disease, this lesion should be present in a child less than two years of age. Over the age of two years, the term oral traumatic granuloma is used [8].

The presence of natal and neonatal teeth is a disturbance of chronology whose etiology is unknown but several factors like superficial position of the tooth germ, infection or malnutrition, hormonal stimulation, febrile states, hereditary transmission of a dominant autosomal gene, hypovitaminosis, congenital syphilis, and osteoblastic activity inside the tooth germ are commonly associated $[9,10]$.

This case report aims to present the occurrence of Riga-Fede Disease and the treatment approach utilized in two cases.

\section{Case 1}

A 2 month old female patient was brought to the Department with the chief complaint of lesion on ventral surface of tongue causing pain and bleeding (Figure 1). The lesion was first noticed one month before by the mother as small erosion, and over time, due to enlargement of the ulceration, significant tissue loss occurred. The mother complained of child exhibiting pain during suckling and even refusing to suck water on occasions, leading to serious dehydration. Clinical intraoral examination revealed whitish tooth like structure in the mandibular anterior region corresponding to 71 . There was a $6 \mathrm{mmX} 5 \mathrm{~mm}$, white, granular, necrotic plaque on the ventral surface of the tongue. On palpation, the area elicited a painful response. The tooth like mass exhibited grade 1 mobility. Based on the clinical findings, the diagnosis of Riga-Fede disease was made.

\section{Treatment}

Since the lesion was extremely painful and due to inadequate nutritional intake by the infant, extraction of the tooth like structure was planned as the treatment of choice.

With parental consent, extraction of the neonatal teeth was carried out under topical local anaesthesia. Gentle curettage of the socket was carried out. The extracted teeth showed a normal morphology, whitish-opaque coronal structures and the presence of root formation (Figure 2).

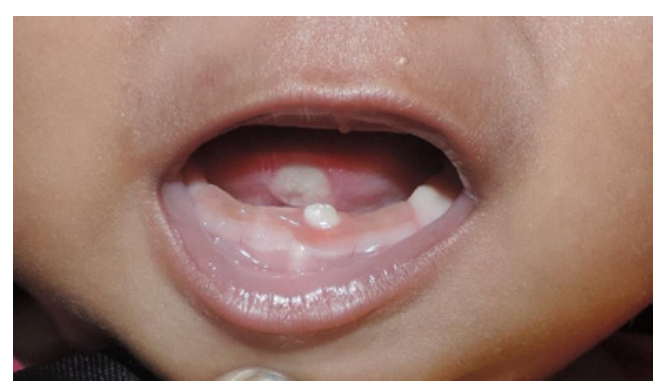

Figure 1: Pre-Operative Photograph Showing Riga Fede Disease.
Austin J Dent - Volume 4 Issue 3 - 2017

ISSN : 2381-9189 | www.austinpublishing group.com

Mehta et al. @ All rights are reserved 


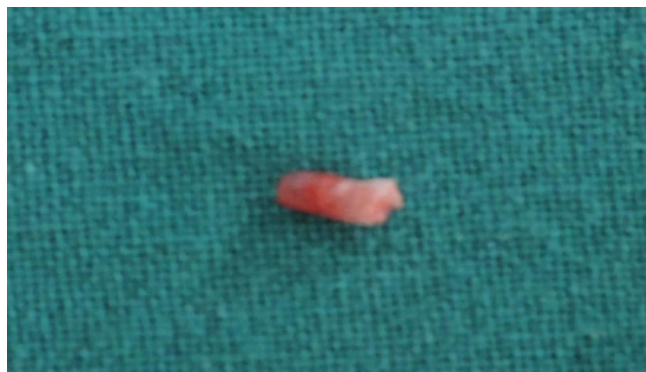

Figure 2: The Extracted Tooth.

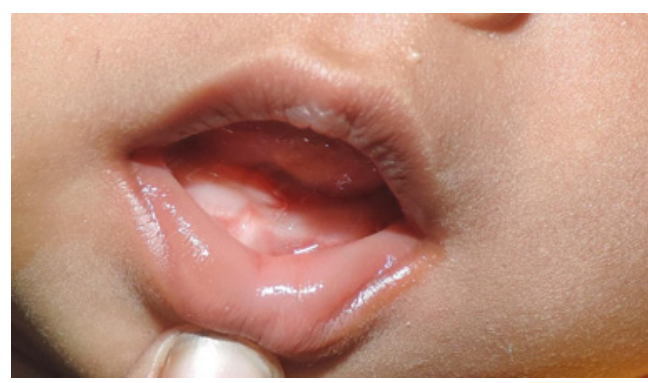

Figure 3: Post-Operative Clinical View After 3 Days.

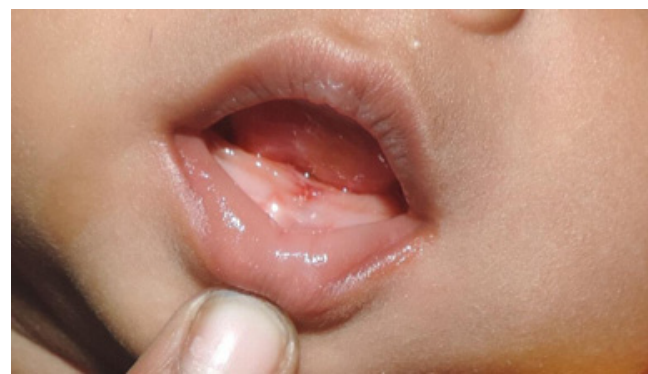

Figure 4: Follow-Up After 3 Weeks.

\section{Outcome and follow-up}

The clinical improvement was evident within 3 days (Figure 3). Within a week, the patient was pain-free and had begun to feed normally. At the end of a three-week follow up period, the ulcer had cicatrized completely (Figure 4).

\section{Case 2}

A 20 days old female patient was brought to the Department with the chief complaint of painful ulceration over the ventral surface of the tongue since 7 days with tooth like structures in the mandibular anterior region (Figure 5). The mother complained of child exhibiting pain during suckling and was not able to nurse the child. Clinical intraoral examination revealed two whitish tooth like structures in the mandibular anterior region corresponding to 71 and 81 (mandibular central incisors). Ventral surface of the tongue showed a single irregularly shaped ulcer, measuring about $6 \mathrm{mmX7} \mathrm{mm}$, extending from the anterior border of the tongue to lingual frenum with a yellowish floor. Based on the clinical findings, the diagnosis of Riga-Fede disease was made.

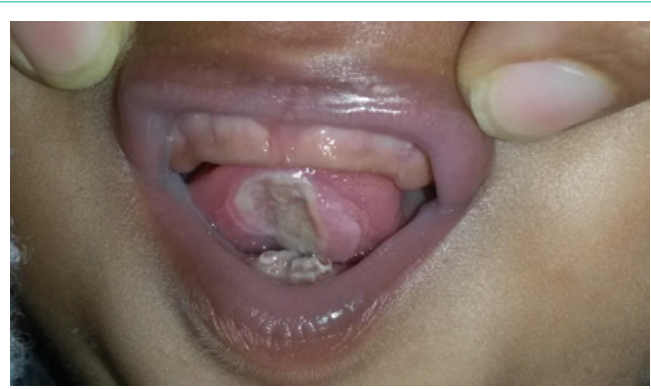

Figure 5: Pre-Operative Photograph Showing Riga Fede Disease.

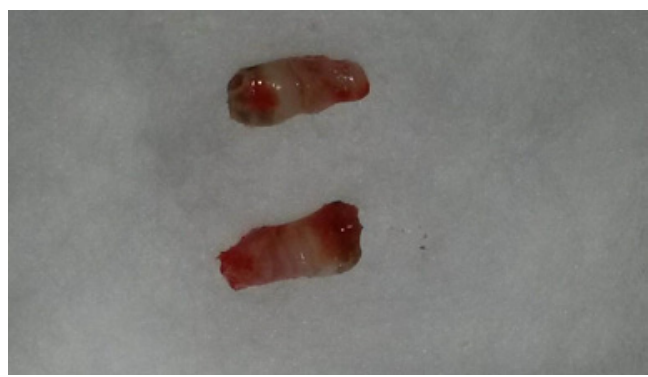

Figure 6: The Extracted Teeth.

\section{Treatment}

The extraction of the neonatal teeth was selected as the treatment of choice for the rapid resolution of the lesion. With parental consent, extraction was carried out under topical local anaesthesia followed by gentle curettage of the socket. The extracted teeth showed a normal morphology, whitish-opaque coronal structures and the presence of root formation (Figure 6).

\section{Discussion}

Riga-Fede disease is a benign ulcerative process of the tongue and frenulum, as a result of repetitive trauma induced by the erupted natal, neonatal or erupting primary mandibular central incisors [2]. It develops due to repetitive trauma of the tongue by natal, neonatal and the eruption of anterior primary teeth during forward and backward movements $[5,6]$. Secondary infection leads to the formation of granulation tissue and the production of a tumor like disc shaped mass showing an ulcerated area in the centre covered with fibrinous pseudomembrane [3]. The ulceration interferes with proper suckling and feeding and puts the neonate at risk for nutritional deficiencies and infants fail to thrive [7].

Riga-Fede disease begins as an ulcerated area with prominent raised edges. With repeated trauma, it may progress to an enlarged, fibrous mass with the appearance of an ulcerative granuloma with superficial necrosis [4]. Histopathologically, it is characterized by an ulcerated mucosa with granulation tissue and inflammatory infiltrate consisting of lymphocytes, macrophages, mast cells and abundant eosinophils $[1,4]$.

In 1983, Elzay coined the term 'traumatic ulcerative granuloma with stromal eosinophilia' (TUGSE) for those chronic ulcerative lesions of the oral mucosa that histopathologically consist mainly of eosinophils [5] Although TUGSE has been mainly reported to occur 
in late adulthood, and not restricted in location to the tongue, it may occur in the buccal mucosa, the vestibule, gingiva, or palate, whereas Riga-Fede disease is almost exclusively restricted to the tongue $[4,5]$.

Riga-Fede disease has also been classified into two discrete groups: 'Early' cases (before 6 months of age) are related to natal or neonatal teeth presenting hypoplastic enamel and underdeveloped roots, with resultant early mobility. 'Late' cases (6 months of age and older) occur with primary dentition and may be related to neurological or developmental disorders like familial dysautonomia, congenital autonomic dysfunction with universal pain loss, Down Syndrome, microcephaly and cerebral palsy $[2,8,9]$.

Several treatments for Riga-Fede disease have been described over the years. The decision of treatment plan is based on factors like: degree of mobility, inconvenience during suckling, interference with breastfeeding, and if the tooth is supernumerary or is part of the normal dentition [11,12]. If these erupted teeth are a part of the primary dentition, maintenance in the mouth is the foremost treatment option except if they become a source of injury. If the teeth are well implanted, they should be left in the arch and conservative treatment should be carried out $[2,13]$. All wright (1958) and Matins, et al. (1998) have recommended smoothening the incisal edges of the teeth to provide a smooth surface to prevent injury to the maternal breast as well as the tongue [2,5,12]. Bjuggren $G$ suggested the use of a feeding splint (1973) while Goho (1996) suggested covering the incisal portion of the tooth with composite resin [12]. But many of natal and neonatal teeth show hypomineralization, and there is limited surface of enamel for bonding of the resin [12]. This, combined with difficulty of adequate moisture control, and limited access may not achieve proper retention of the composite leading to restorative failure $[1,12]$ The use of analgesic gels to relieve pain and aid in wound healing can also accelerate the healing process [2].

When the conservative methods fail to resolve the lesion and impede with feeding resulting in the child being extremely dehydrated or malnourished, or when they are extremely mobile with a risk of aspiration, extraction of natal teeth is indicated $[3,6]$. If extraction is required, it should not cause any difficulties as these teeth can be removed with a forceps or even with the fingers [12]. The extraction can be delayed till the child is 10 days of age or more for appropriate blood levels of vitamin K [9]. During this waiting period commensal flora of the intestine will be established to produce vitamin K, which is necessary for the production of prothrombin in the liver [13]. If it is not possible to wait then it is advisable to evaluate the need for administration of vitamin $\mathrm{K}$ with a pediatrician, if the newborn was not medicated with vitamin $\mathrm{K}$ immediately after birth $[9,13]$. Vitamin $\mathrm{K}(0.5-1.0 \mathrm{mg})$ is administered intramuscularly to the baby as part of immediate medical care to prevent hemorrhagic disease of the newborn $[9,11]$. Once extraction is carried out, the underlying dental papilla and Hertwig's epithelial root sheath should be removed during the extraction to prevent the development of root structure that could continue if these structures are left in situ [12].

The early diagnosis and treatment of Riga-Fede disease is imperative to avoid extensive periods of pain and possible dehydration $[2,5,13]$. The child must be monitored to resolve the function and aesthetics of the normal primary dentition [14].

\section{References}

1. Yacovone L, Roberson D, Wai M. Riga-Fede Disease: A Rare Sublingual Traumatic Ulcerative Lesion in a Child.Otolaryngol Head Neck Surg. 2011; 146: 333-334.

2. Bafna $Y$, Khandelwal V, Bafna M, Nayak PA. Management of sublingual ulceration in a 12-month-old child. BMJ Case Rep. 2013.

3. Parisay I, Ghafournia M, Shafagh M, Mousavi SA. Lingual Traumatic Ulceration (Riga-Fede Disease): Report of a Case and Review. J Dent Mater Tech. 2013; 2: 142-147.

4. Van der Meij EH, De Vries TW, Eggink HF, De Visscher JG. Traumatic lingual ulceration in a newborn: Riga-Fede disease. Ital J Pediatr. 2012; 38: 1-5

5. Costacurta M, Maturo P, Docimo R. Riga-fede disease and neonatal teeth Oral Implantol. 2012; 5: 26-30.

6. Slayton RL. Treatment alternatives for sublingual traumatic ulceration (RigaFede disease). Pediatr Dent. 2000; 22: 413-414.

7. Bhatt R, Dave B, Sheth M. Riga-Fede Disease: Report of a Case with Literature Review. Adv Hum Biol. 2015; 5: 52-55.

8. Machuca G, Rodríguez S, Vargas MP, Suárez C, Bullón P. Management of Riga-Fede disease: A case report. Journal of Disability and Oral Health. 2007; 8: 28-30.

9. Kumar PPS, Dhull KS, Dhull RS, Panda S, Yadav S, Indira MD. Riga-Fede Syndrome: Review of Literature with report of three Cases. International Journal of Oral and Maxillofacial Pathology. 2013; 4: 40-44.

10. Mhaske S, Yuwanati MB, Mhaske A, Ragavendra R, Kamath K, Saawarn S. Natal and Neonatal Teeth: An Overview of the Literature. ISNR Pediatrics. 2013.

11. Malki GA, Al-Badawi EA, Dahlan MA. Natal Teeth: A Case Report and Reappraisal. Case Reports in Dentistry 2015

12. Ardeshana A, Bargale S, Karri A, Dave B. Dentitia Praecox-Natal Teeth: A Case Report and Review. Journal of Applied Dental and Medical Sciences. 2016; 2: 44-51.

13. Volpato LER, Simões CAD, Simões F, Nespolo PA, Borges AH. Riga-Fede Disease Associated with Natal Teeth: Two Different Approaches in the Same Case. Case Reports in Dentistry. 2015.

14. Deep SB, Ranadheer E, Rohan B. Riga-Fede Disease: Report of a case with Literature Review. J Academy Adv Dental Research. 2011; 2: 27-30.
Austin J Dent - Volume 4 Issue 3 - 2017 ISSN : 2381-9189 | www.austinpublishing group.com Mehta et al. () All rights are reserved
Citation: Mehta A, Chaudhary S, Chaitra TR and Sinha A. Riga-Fede Disease (Dentitia Praecox): Report of Two Cases with Literature Review. Austin J Dent. 2017; 4(3): 1073. 